\title{
Transport in dimerized and frustrated spin systems
}

\author{
F. Heidrich-Meisner ${ }^{\mathrm{a} *}$, A. Honecker ${ }^{\mathrm{a}}$, D.C. Cabra ${ }^{\mathrm{b}}$ and W. Breniga \\ anstitut für Theoretische Physik, Technische Universität Braunschweig, \\ Mendelssohnstrasse 3, 38106 Braunschweig, Germany \\ ${ }^{\text {b}}$ Laboratoire de Physique, Ecole Normale Superieure de Lyon, \\ 46, Allée d'Italie, 69364 Lyon, France
}

\begin{abstract}
We analyze the Drude weight for both spin and thermal transport of one-dimensional spin- $1 / 2$ systems by means of exact diagonalization at finite temperatures. While the Drude weights are non-zero for finite systems, we find indications of a vanishing of the Drude weights in the thermodynamic limit for non-integrable models implying normal transport behavior.
\end{abstract}

Transport properties of one-dimensional spin$1 / 2$ systems have recently attracted strong interest from the theoretical side (see e.g. 123 , 456] and references therein). An intensively studied issue is the question under which conditions ballistic transport occurs at zero frequency and finite temperature $T>0$, characterized by a non-zero Drude weight $D$. This quantity is the zero-frequency weight of the real part of the conductivity $\sigma(\omega)$, namely $\operatorname{Re} \sigma(\omega)=D(T) \delta(\omega)+$ regular part. While it is known that the anisotropic Heisenberg chain has a finite Drude weight for thermal transport since the energy-current operator is a conserved quantity [7], there is a controversial discussion [2, 345. whether transport remains ballistic if the model is extended by dimerization or frustration.

In this paper, we will focus on two examples, namely thermal transport of dimerized chains and spin transport in frustrated chains. We consider the Hamiltonian $(S=1 / 2)$

$H=\sum_{l=1}^{N} h_{l}=J \sum_{l=1}^{N}\left[\lambda_{l} \vec{S}_{l} \cdot \vec{S}_{l+1}+\alpha \vec{S}_{l} \cdot \vec{S}_{l+2}\right]$.

Here, $N$ is the number of sites and $h_{l}$ is the local energy density. We set $\lambda_{l}=1$ if $l$ is even and $\lambda_{l}=\lambda$ otherwise. Note that we use periodic

${ }^{*}$ Corresponding author. Tel.: +49-531-391-5184. E-mail: f.heidrich-meisner@tu-bs.de. boundary conditions.

The thermal Drude weight $D_{\text {th }}(T)$ can be obtained from [7] $\left(p_{n}=e^{-E_{n} / T} / Z ; Z=\sum_{n} e^{-E_{n} / T}\right)$

$D_{\mathrm{th}}(T)=\frac{\pi}{N T^{2}} \sum_{\substack{m, n \\ E_{m}=E_{n}}} p_{n}\left|\left\langle m\left|j_{\mathrm{th}}\right| n\right\rangle\right|^{2}$

while the Drude weight $D_{\mathrm{s}}(T)$ for spin transport follows from 89

$D_{\mathrm{s}}(T)=\frac{\pi}{N}\left[\langle-\hat{T}\rangle-2 \sum_{\substack{m, n \\ E_{m \neq n} \neq E_{n}}} p_{n} \frac{\left|\left\langle m\left|j_{\mathrm{s}}\right| n\right\rangle\right|^{2}}{E_{m}-E_{n}}\right]$.

$j_{\text {th }}$ and $j_{\mathrm{s}}$ denote the energy- and spin-current operator, respectively. They obey equations of continuity: $i\left[H, h_{l}\left[S_{l}^{z}\right]\right]=-\left(j_{\mathrm{th}[\mathrm{s}], l+1}-j_{\mathrm{th}[\mathrm{s}], l}\right)$ with $j_{\operatorname{th}[\mathrm{s}]}=\sum_{l} j_{\operatorname{th}[\mathrm{s}, l, l}$. The local energy density $h_{l}$ is defined in Eq. (11) and $S_{l}^{z}$ is the local magnetization density. We refer the reader to Refs. 310] for full expressions of $j_{\mathrm{th}[\mathrm{s}]}$. Both current operators do not commute with $H$ as soon as $\lambda \neq 1$ or $\alpha \neq 0$. The operator $\hat{T}=$ $J \sum_{l}\left[\lambda_{l} S_{l}^{+} S_{l+1}^{-}+4 \alpha S_{l}^{+} S_{l+2}^{-}+\right.$H.c. $]$is the kinetic energy 810 .

Now we turn to the discussion of our numerical results for the thermal Drude weight $D_{\text {th }}(T)$ of dimerized chains which we obtain by complete diagonalization of $H$. The data are shown in the main panel Fig. 1(a) for $N=8, \ldots, 16$ sites. Since finite-size effects are strongest at low tem- 
peratures, we concentrate on $T>J$. Here, the leading contributions to $D_{\mathrm{th}}(T)$ take the form

$D_{\mathrm{th}}(T)=C_{\mathrm{th}, 1} / T^{2}+C_{\mathrm{th}, 2} / T^{3}+\ldots$.

While we compute $C_{\mathrm{th}, 1}$ directly by setting $Z=$ $2^{N}$ and $e^{-\beta E_{n}}=1$ in Eq. (2), $C_{\mathrm{th}, 2}$ is extracted by a fit of Eq. (44) to the numerical data at high T. As can be seen in the inset of Fig. 1(a), both coefficients exhibit a significant decrease with system size though not monotonically. The system sizes are too small to draw conclusions about the actual finite-size dependence of $D_{\mathrm{th}}$, but the observed behavior does not support the notion of a finite Drude weight for $N \rightarrow \infty$ contrasting a recent claim of Ref. [2].

The second example is spin transport of frustrated chains in the gapped regime. In the main panel of Fig. प(b), we show $D_{\mathrm{s}}(T)$ for $\alpha=0.35$ and even $N \leq 18$. The main features are a finite Drude weight at $T=0$ and a monotonic decrease of $D_{\mathrm{s}}(T)$ for $T>0.5 J . D_{\mathrm{s}}(T=0)>0$ has also been reported in Ref. [10] for $\alpha<0.5$ and $N \leq 20$. Extension to larger systems at $T=0$ (e.g. with Lanczos techniques) should clarify whether $D_{\mathrm{s}}(T=0)>0$ is a property that survives in the thermodynamic limit $N \rightarrow \infty$.

We further concentrate on the analysis at large $T$, namely the high-temperature prefactor $C_{\mathrm{s}}=$ $\lim _{T \rightarrow \infty}\left[T \cdot D_{\mathrm{s}}(T)\right]$. Its finite-size dependence is illustrated in the inset of Fig. (1) for $\alpha=0.35,1$ and $N=8,9, \ldots, 18$. Apart from odd-even finite-size effects, which are more pronounced for $\alpha=0.35$, both data sets exhibit a monotonic decrease of $C_{\mathrm{s}}$ with system size, as it is particularly obvious in the case of $\alpha=1$. This provides strong evidence for $D_{\mathrm{s}} \rightarrow 0$ for $N \rightarrow \infty$ in the gapped regime of frustrated chains which is consistent with Ref. [11.

In summary, we have numerically studied the thermal Drude weight of dimerized chains and the spin Drude weight of frustrated chains at $T>0$. The finite-size analysis supports the conclusion of a zero Drude weight in the thermodynamic limit in these examples. We have found analogous results for both kinds of transport for the models discussed here and spin ladders which will be reported in detail elsewhere 12.

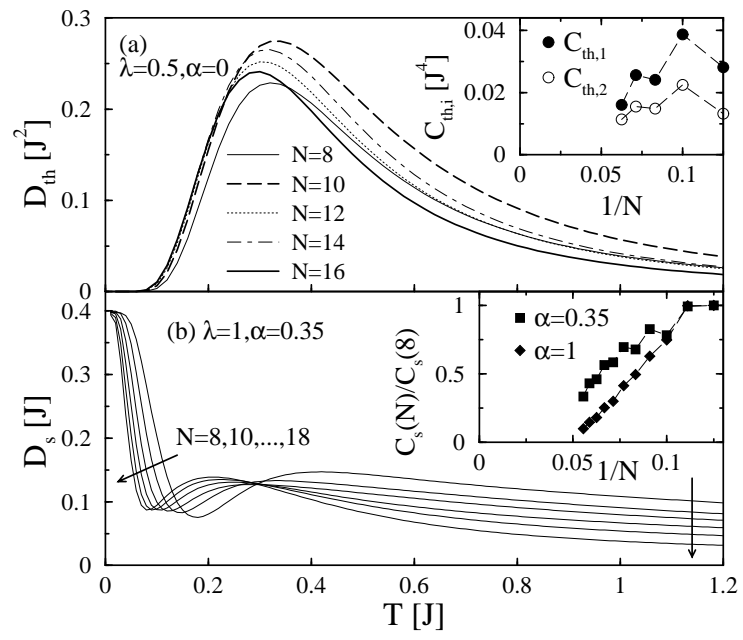

Figure 1. (a) Main panel: Thermal Drude weight $D_{\text {th }}(T)$ of a dimerized chain with $\lambda=0.5$ (see Eq. (11)). Inset: $C_{\mathrm{th}, i}, i=1,2$ vs. $1 / N$ (see text). (b) Main panel: Drude weight $D_{\mathrm{s}}(T)$ of a frustrated chain with $\alpha=0.35$. Arrows indicate increasing $N$. Inset: $C_{\mathrm{s}}$ vs. $1 / N$ for $N=8,9, \ldots, 18$.

\section{REFERENCES}

1. A. Klümper and K. Sakai, J. Phys. A 35 (2002) 2173.

2. J. V. Alvarez and C. Gros, Phys. Rev. Lett. 89 (2002) 156603.

3. F. Heidrich-Meisner et al., Phys. Rev. B 66 (2002) 140406(R); Phys. Rev. Lett 92 (2004) 069703.

4. K. Saito, Phys. Rev. B 67, (2003) 064410.

5. E. Orignac et al., Phys. Rev. B 67 (2003) 134426.

6. S. Fujimoto and N. Kawakami, Phys. Rev. Lett. 90 (2003) 197202.

7. X. Zotos et al., Phys. Rev. B 55 (1997) 11029.

8. B.S. Shastry and B. Sutherland, Phys. Rev. Lett.'65 (1990) 243.

9. H. Castella et al., Phys. Rev. Lett. 74 (1995) 972.

10. J. Bonča et al., Phys. Rev. B 50 (1994) 3415.

11. A. Rosch and N. Andrei, Phys. Rev. Lett. 85 (2000) 1092.

12. F. Heidrich-Meisner et al., Phys. Rev. B 68 (2003) 134436. 